\title{
Comparison of Pneumatic, Ultrasonic and Combination Lithotripters in Percutaneous Nephrolithotripsy
}

\author{
Kursad Zengin ${ }^{1}$, Nevzat Can Sener², Okan Bas ${ }^{3}$, Ismail Nalbant ${ }^{4}$, Inan Alisir ${ }^{5}$ \\ ${ }^{1}$ Department of Urology, Bozok University, Faculty of Medicine, Yozgat, Turkey; ${ }^{2}$ Department of Urology, \\ Numune Education and Research Hospital, Adana, Turkey; ${ }^{3}$ Department of Urology, Abdurrahman \\ Yurtaslan Education and Research Hospital, Ankara, Turkey; ${ }^{4}$ Department of Urology, Yenimahalle State \\ Hospital, Ankara, Turkey; ${ }^{5}$ Department of Urology, Fethiye State Hospital, Mugla, Turkey
}

\section{ABSTRACT}

Purpose: We aimed to compare the outcomes of pneumatic (PL), ultrasonic (UL) and combined (PL/UL) lithotripsy performed in percutaneous lithotripsy (PNL) according to success rates and stone clearence.

Materials and Methods: The medical records of 512 patients treated with PNL between April 2010 and April 2013 were evaluated. Postoperative stone analysis revealed as calcium oxalate in 408 of these patients. The operation notes of 355 patients recorded in detail with complete parameters were reviewed. According to stone disintegration method, patients were divided into three groups: PL only in Group I, UL only in Group II, and UL/PL combination in Group III. Number of patients was 155, 110 and 90, respectively.

Results: Fluoroscopy screening time was significantly shorter in group II, and III compared to group I ( $\mathrm{p}<0.001)$. The failure rates were 13.5\% (21 patients) for group I, 3.6\% (4 patients) for group II, and 3.3\% (3 patients) for group III. There was a significant statistical difference in favor of group II and III by means of success ( $p=0.023)$. Group II and III had larger FSA, and this was statistically significant ( $\mathrm{p}=0.032)$. Stone disintegration time (SDT) was $64.0 \pm 41.92$ minutes for group I, $49.5 \pm 34.63$ for group II, and $37.7 \pm 16.89$ for group III. Group III has a statistically significant shorter SDT ( $p=0.011)$. Conclusions: We concluded that, in cases with high stone burden, where faster and efficient lithotripsy is needed, combined ultrasonic / pneumatic lithotripter may be the ideal choice and in suitable cases ultrasonic lithotripter usage provides important advantages to the surgeon.

\section{ARTICLE INFO}

\section{Key words:}

Percutaneous; Operative Time; Calculi

Int Braz J Urol. 2014; 40: 650-5

Submitted for publication: November 05, 2013

Accepted after revision:

May 03, 2014

\section{INTRODUCTION}

Even though open surgery had been the first treatment option for most kidney stones about 30 years ago, recent technological advancements made endourology more feasible and accessible. Current European Urology Guidelines recommend Percutaneous Nephrolithotomy (PNL) for kidney stones $>2 \mathrm{~cm}(1)$.
Primary goal for kidney stone treatment is to remove as much stone volume as possible. Remaining stones may cause pain, infection and/or obstruction, as well as work as a seed for new stone formation (2). Flexible (Electrohydraulic or laser), rigid pneumatic (PL), and ultrasonic (UL) lithotripters are the current alternatives for stone disintegration in PNL. Various advantages and disadvantages of these lithotripters have been shown on stone free rates (3). 
In this study, we aimed to compare the outcomes of PL, UL and combined (PL/UL) lithotripsy performed in PNL in our clinic according to success rates and stone clearance.

\section{PATIENTS AND METHODS}

\section{Patients}

The medical records of 512 patients treated with PNL at urology department of Diskapi Education and Research Hospital between April 2010 and April 2013 were evaluated. Postoperative stone analysis revealed calcium oxalate in 408 of these patients. Retrospectively, the operation notes of 355 patients recorded in detail with complete parameters were reviewed. According to stone disintegration method, patients were divided into three groups: PL only in Group I, UL only in Group II, and UL/PL combination in Group III. Number of patients was 155, 110 and 90, respectively.

All patients had been assessed by complete blood count, kidney and liver function tests, serum electrolytes, coagulation parameters, hepatic markers, urinalysis and urine cultures before surgery. Patients with urinary tract infections and/or coagulation disorders received appropriate treatment before the operation.

All patients were evaluated with computed tomography before the operation. Stone burden was calculated (area) by multiplying the longest length with the perpendicular length in millimeter square unit $\left(\mathrm{mm}^{2}\right)$. When dealing with multiple stones, all stones were measured individually and the sum was used. Total operation time (OT) and stone disintegration time (SDT) were measured separately. SDT was accepted as the overall process for stone fragmentation and extraction. In all cases, a complete stone removal was the main target for treatment. Stone area before and after the operation was assessed and the difference gave the Fragmented Stone Area (FSA). FSA was divided to SDT and stone clearance $\left(\mathrm{mm}^{2}\right)$ minute) was measured for all individual patients.

PNL technique

All PNL procedures were performed under general anesthesia. At the beginning of the proce- dure, a 5F open-ended ureteral catheter was inserted into the ipsilateral ureter in the lithotomy position. The distal part of catheter was fixed to a $16 \mathrm{~F}$ Foley bladder catheter. The patient was then placed in the prone position, and percutaneous access was achieved under C-arm fluoroscopy guidance using an 18-gauge needle and guidewire. The nephrostomy tract was dilated up to $30 \mathrm{~F}$ by Amplatz dilators (Boston Scientific, Boston, USA), and an Amplatz sheath (30F) was placed. Stone fragmentation was accomplished by using a pneumatic lithotripter or ultrasonic lithotripter (Lithoclast; EMS, Nyon, Switzerland). All operations were performed by the same surgical team. The decision of lithotripter choice was done by the chief surgeon in the operation. At the end of the operation, a Malecott re-entry catheter was placed in all patients. Patients were evaluated with urinary ultrasound (US) and computed tomography (CT) one month after the surgery.

Patients with asymptomatic stones (smaller than $3 \mathrm{~mm}$ and non-obstructive) were considered as clinically insignificant urolithiasis. Treatment results were classified as "stone-free (SF)", "clinically insignificant residual fragments (CIRF)" and "failed" (in the presence of residual stones $\geq 3 \mathrm{~mm}$ ). Patients who were stone free or with clinically insignificant urolithiasis at the end of the procedure were considered as successful.

\section{Statistical analysis}

Statistical analyses were performed using the Statistical Package for Social Sciences version 11.5 software (SPSS Inc, Chicago, IL, United States). Continuous measurement was investigated by Shapiro Wilk test for normal distribution of variables while continuous measurement variables were presented by mean \pm standard deviations (SDs) [median (minimum-maximum)], nominal variables were presented by the number of cases and percentage (\%). Variables were assessed by Pearson's chi-square test, One-Way ANOVA and Kruskal Wallis tests. A value of $p<0.05$ was considered as statistically significant.

\section{RESULTS}

There were 213 (60\%) men and 142 (40\%) women. Mean patient age was $45.2 \pm 14.2$ years in 
group I; $43.7 \pm 13.75$ years in group II and $45.1 \pm 14.46$ years in group III. The mean stone burden was $846.9 \pm 400.53 \mathrm{~mm}^{2}$ in group I; $902.7 \pm 705.32 \mathrm{~mm}^{2}$ in group II and $910.4 \pm 804.47 \mathrm{~mm}^{2}$ in group III. The patient demographics are presented in Table-1.

Mean hospital stay was $4.3 \pm 4.14$ days in group I; $3.5 \pm 2.65$ days in group II and $2.3 \pm 2.59$ days in group III. There was a statistically significant difference by means of hospital stay favoring group III ( $\mathrm{p}=0.006)$. Mean fluoroscopy screening time was $2.6 \pm 3.24,1.0 \pm 2.23$ and $1.3 \pm 1.44$ minutes in group I, group II and group III, respectively. Fluoroscopy screening time was significantly shorter in groups II and III compared to group I $(\mathrm{p}<0.001)$. Operative and postoperative data are detailed in Table- 2 .

105 patients $(67.7 \%)$ in group I, 89 (80.9\%) in group II, and 77 (85.5\%) in group III were SF after the operation. There were 29 patients (18.7\%) in group I, 17 patients (15.4\%) in group II, and 10 patients (11.1\%) in group III classified as CIRF after the operation. The failure rates were 13.5\% (21 patients) for group I, 3.6\% (4 patients) for group II, and 3.3\% (3 patients) for group III. There was a significant statistical difference in favor of groups II and III by means of success $(p=0.023)$. The patients with residual fragments $\geq 3 \mathrm{~mm}$ were either followed-up without treatment or retreated with shock wave lithotripsy (SWL), re-PNL, or flexible URS according to clinical significance. The details are summarized in Table-3.

FSA was found as $730.0 \pm 335.71 \mathrm{~mm}^{2}$ for group I, $831.3 \pm 604.24 \mathrm{~mm}^{2}$ for group II, and $882.0 \pm 740.21 \mathrm{~mm}^{2}$ for group III. Group II and III had larger FSA, and this was statistically significant ( $\mathrm{p}=0.032$ ). SDT was $64.0 \pm 41.92$ minutes for group I, $49.5 \pm 34.63$ for group II, and $37.7 \pm 16.89$ for group III. Group III has a statistically significant shorter SDT $(p=0.011)$. Stone clearance was $11.9 \pm 12.38$ for PL (group I), $19.1 \pm 13.56$ for UL (group II), and $24.9 \pm 21.38$ for UL + PL (group III). UL alone (group II), and UL + PL (group III) had longer stone clearance. The results of these groups had a significant statistical difference over group I ( $p=0.027)$.

The majority of complications that needed intervention were bleeding and urinary leakage after the removal of nephrostomy tube. There were no statistically significant differences between the three groups in terms of blood transfusions $(\mathrm{p}=0.582)$.

Four patients $(2.5 \%)$ were treated with a double-J stent for prolonged urinary leakage after removal of the nephrostomy tube in group I. Four patients $(2.5 \%)$ in group I, 2 patients $(1.8 \%)$ in group II and 2 patients $(2.2 \%)$ in group III were treated with semirigid ureteroscopy for steinstrasse.

Table 1 - Demographical data of patients according to groups.

\begin{tabular}{|c|c|c|c|c|}
\hline Variables & Group I $(n=155)$ & Group II $(n=110)$ & Group III $(n=90)$ & $\mathrm{p}$ value \\
\hline Mean age \pm SD (years) & $45.1 \pm 14.20$ & $43.7 \pm 13.75$ & $45.1 \pm 14.46$ & $0.838^{\mathrm{a}}$ \\
\hline Gender (n) & & & & $0.058^{b}$ \\
\hline Male & $98(\% 63.2)$ & $52(\% 47.2)$ & $63(\% 70)$ & \\
\hline Female & $57(\% 36.8)$ & $58(\% 52.8)$ & $27(\% 30)$ & \\
\hline Mean stone burden ( $\left.\mathrm{mm}^{2}\right)$ & $846.9 \pm 400.53$ & $902.7 \pm 705.32$ & $910.4 \pm 804.47$ & $0.17^{\mathrm{a}}$ \\
\hline Previous renal intervention(s) & & & & $0.190^{b}$ \\
\hline Primary & $120(\% 77.4)$ & $94(\% 85.4)$ & $58(\% 64.4)$ & \\
\hline Secondary & $26(\% 16.7)$ & $12(\% 10.9)$ & $28(\% 31.1)$ & \\
\hline Tertiary & $9(\% 5.8)$ & $4(\% 3.6)$ & $4(\% 4.4)$ & \\
\hline
\end{tabular}

${ }^{a}$ One-Way Analysis of Variance

b Pearson's chi-square test 
Table 2 - Comparison of operative and postoperative data.

\begin{tabular}{lcccc}
\hline Variables & Group I & Group II & Group III & $p$ \\
\hline Hospital stay (days) & $4.3 \pm 4.14$ & $3.5 \pm 2.65$ & $2.3 \pm 2.59$ & $0.006^{\mathrm{c}}$ \\
Nephrostomy removal time (days) & $3.0 \pm 2.98$ & $2.3 \pm 0.96$ & $2.2 \pm 0.65$ & $0.141^{\mathrm{c}}$ \\
Mean fluoroscopy time (min) & $2.6 \pm 3.24$ & $1.0 \pm 2.23^{\dagger}$ & $1.3 \pm 1.44^{\dagger}$ & $<0.001^{\mathrm{c}}$ \\
Blood transfusion & $31(\% 20)$ & $23(\% 20,9)$ & $15(\% 16.7)$ & $0.582^{\mathrm{b}}$ \\
Fragmented stone area (fsa) & $730.0 \pm 335.71$ & $831.3 \pm 604.24 \dagger$ & $882.0 \pm 740.21 \dagger$ & $0.032^{\mathrm{b}}$ \\
Fragmentation area ratio (\%) & $86.6 \pm 21.56$ & $95.7 \pm 13.52 \dagger$ & $98.3 \pm 5.55 \dagger$ & $0.040^{\mathrm{b}}$ \\
Total operation time (min.) & $103.7 \pm 48.30$ & $86.7 \pm 40.56 \dagger$ & $75.8 \pm 23.00 \dagger$ & $0.006^{\mathrm{b}}$ \\
Stone disintegration time (sdt) (min.) & $64.0 \pm 41.92$ & $49.5 \pm 34.63$ & $37.7 \pm 16.89 \ddagger$ & $0.011^{\mathrm{b}}$ \\
Stone clearance (fsa/sdt) & $11.9 \pm 12.38$ & $19.1 \pm 13.56 \dagger$ & $24.9 \pm 21.38 \dagger$ & $0.027^{\mathrm{b}}$ \\
Stone-free rate (n) & $105(\% 67.7)$ & $89(\% 80.9)$ & $77(\% 85.5)$ & 0.037 \\
CIRF (n) & $29(\% 18.7)$ & $17(\% 15.4)$ & $10(\% 11.1)$ & 0.028 \\
Failed (n) & $21(\% 13.5)$ & $4(\% 3.6) \dagger$ & $3(\% 3.3) \dagger$ & 0.023 \\
SC/AC & $134(\% 86.4)$ & $106(\% 96.3)$ & $87(\% 96.6)$ & 0.646 \\
\hline
\end{tabular}

${ }^{a}=$ Kruskal-Wallis test; ${ }^{b}=$ Pearson's chi-square test; ${ }^{c}=$ Kruskal-Wallis test; ${ }^{\dagger}=$ Statistically significant difference between groups II-III and I $(p<0.05)$;

${ }^{\ddagger}=$ Statistically different group from others $(\mathrm{p}<0.05) ; \mathrm{CIRF}=$ Clinically insignificant residual fragments $(<4 \mathrm{~mm}) ; \mathrm{SC} / \mathrm{AC}=$ Successful cases (Stone free $\left.+\mathrm{CIRF}\right)$ rate in all cases

Table 3 - The procedures applied for patients with residual fragments $\geq 3 \mathrm{~mm}$ after the operation.

\begin{tabular}{lccc}
\hline Variables & Group I $(\mathrm{n}=155)$ & Group II $(\mathrm{n}=110)$ & Group III $(\mathrm{n}=90)$ \\
\hline $\begin{array}{l}\text { Number of patients with residual } \\
\text { fragments } \geq 3 \mathrm{~mm} \text { after PNL }\end{array}$ & $21(13.5 \%)$ & $4(3.6 \%)$ & $3(3.3 \%)$ \\
Follow-up without treatment & $6(28.6 \%)$ & $2(50 \%)$ & $1(33.3 \%)$ \\
SWL & $7(33.4 \%)$ & 0 & $1(33.3 \%)$ \\
Re-PNL & $4(19 \%)$ & $1(25 \%)$ & 0 \\
Flexible URS & $4(19 \%)$ & $1(25 \%)$ & $1(33.3 \%)$ \\
\hline
\end{tabular}

\section{DISCUSSION}

Published studies had shown that combined pneumatic / ultrasonic lithotripter is an effective and safe method to use $(4,5)$. However, there are few studies comparing the combined ultrasound / pneumatic lithotripter to only pneumatic or only ultrasonic lithotripter (6-8).

Pietrow et al. randomized 20 patients, who underwent PNL, into 2 groups. He applied only ul- trasonic lithotripsy to one group and combined ultrasonic / pneumatic lithotripsy to the other group. It was found that stone disintegration time was significantly longer in only ultrasonic lithotripsy cases (43.7 minutes compared to $21.1, \mathrm{p}=0.036$ ); on the contrary, stone clearance rate was lower in standard ultrasonic device than the combined pneumatic/ ultrasonic lithotripter $\left(16.8 \mathrm{~mm}^{2} / \mathrm{min}\right.$ compared to 39.5 $\mathrm{mm}^{2} / \mathrm{min}, \mathrm{p}=0.028$ ). Differences of stone free and success rates between the two groups were not sig- 
nificant (6). In an in-vitro study by Auge et al., combined device was found more effective in stone breaking and removing than both standard pneumatic and ultrasonic devices. The average time was determined as 7.41 minutes for combination device, 12.87 minutes for ultrasonic device, and 23.76 minutes for pneumatic device $(p<0.003)$. When dimensions of the removed fragments were compared, combination device was superior to the other two devices on fragmentation. Average fragment size measure was 1.67 $\mathrm{mm}$ in combination device, $3.67 \mathrm{~mm}$ in ultrasonic device, and $9.07 \mathrm{~mm}$ in pneumatic device. Ultrasonic device generated smaller fragments and created better results than pneumatic device (9).

Agras et al. conducted a prospective, randomized study, comparing effectiveness of pneumatic and combined pneumatic/ultrasonic device, and found stone-free rates of 52.3\% and 70.7\%, in pneumatic and combination groups respectively. Taking into account clinically insignificant residual stones, these rates were $81.8 \%$ and $78 \%$, in pneumatic and combination groups respectively. Although there was a difference between the two groups in terms of both stone free and success rates, there was no statistical difference. This was attributed to small number of cases. Residual stone rates for clinically insignificant and smaller than $3 \mathrm{~mm}$ in diameter were $29.5 \%$ and $7.3 \%$, in only pneumatic device group and combination device group, respectively $(\mathrm{p}=0.005)$. Attention was drawn to the significant difference and it was stated that combination device was better in removing clinically insignificant residual stones (10). Karakan et al. designed a study comparing lithotripters on 227 consecutive cases undergoing PNL. They revealed better stone free rates in favor of UL group (7). We found similar success rates for UL and combination, all better than PL group. This was attributed to the aspiration ability of the ultrasonic probe for floating fragments. Also, removal of broken stone fragments process was frequently repeated in pneumatic probe usage.

In our study, when total operative time and stone clearance rates are taken into account, total operative time was longer in Group I than Groups II and III. Stone disintegration was faster in group III and stone clearance was worse in Group 1 than the other groups. The success in ultrasonic lithotripter can be attributed to preference of ultrasonic litho- tripsy in cases of endurable stones and usage of oscillating type ultrasonic lithotripter. In our clinical experience, we observed that oscillating type ultrasonic lithotripter was more effective than stationary ultrasonic lithotripter in terms of stone fragmentation. Even though its aspiration ability is poorer, when compared to stationary ultrasonic lithotripter, this cannot be considered a total disadvantage. For removing the broken fragments, surgeon has to remove all fragments manually when using PL; therefore this may cause prolongation of total surgery and stone disintegration time.

Tepeler et al. analyzed the parameters of factors affecting fluoroscopy time. They reported a mean of $10.19 \pm 0.3$ minutes of screening. They revealed stone size and number of accesses were important factors for fluoroscopy time (11). This seems higher than our fluoroscopy times but they have higher stone burden than our cohort. They did not compare lithotripters and outcomes. When we analyzed our outcomes, fluoroscopy screening was longer Group I when compared to Group II and Group III. We believe surgeon's need to remove each fragment following fragmentation with PL was the main reason behind that matter.

In an in-vitro study conducted by Auge et al., biggest stone fragments fragmented by different lithotripters were compared. When biggest removed fragments were compared, combination device was found to be superior to other two devices. Average particle sizes were $1.67 \mathrm{~mm}, 3.67 \mathrm{~mm}$, and 9.07 $\mathrm{mm}$ in combination device, ultrasonic device, and pneumatic device, respectively. Ultrasonic device had better results than pneumatic device in forming smaller particles (9). When the results were compared by means of failure and CIRF, group I had higher numbers. We believe the usage of ultrasonic probe and its ability to create smaller fragments facilitated higher stone free rates and smaller fragment sizes of Group II and Group III. According to stone sizes, different approaches can be used for residual stones (12). Because of higher failure rates observed in group I (13.5\%), 7 patients underwent SWL, 4 patients underwent re-PNL, and 4 patients were operated with flexible URS for residual fragments. The failure rates were 3.6\% in group II, and 3.3\% in group III. Naturally re-intervention rates were lower for these groups. 
Mean hospital stay for PNL is usually short. However, complications are the main reason for prolonged hospital stay $(13,14)$. In our study length of hospital stay was $4.3 \pm 4.14$ days in Group 1 and $2.3 \pm 2.59$ in Group 3. This can be attributed to the longer operative times as well as higher rates of transfusion needs in Group I, and this was statistically significant $(p=0.006)$. The longer duration of operations may be due to multiple stone extractions with forceps in Group 1, while in the Group 3, ultrasonic lithotripter also aspirates the fragmented stones simultaneously.

There are several limitations of this study. It is a retrospective study and patients were not randomized. Also, laser use was not compared. However, with the largest cohort comparing the parameters in treatment with different lithotripters, we believe this study yields important results.

\section{CONCLUSIONS}

Combined ultrasonic / pneumatic probe disintegrates stones faster and this shortens total operative time. Therefore, stone clearance is faster. Even though no statistically significant success rate difference between groups was detected, best results were achieved in ultrasonic probe groups (Groups II and III). Faster and more successful operations resulted in shorter fluoroscopy time and shorter hospital stay.

We concluded that, in cases with high stone burden, where faster and efficient lithotripsy is needed, combined ultrasonic / pneumatic lithotripter may be the ideal choice and in suitable cases ultrasonic lithotripter usage provides important advantages to the surgeon.

\section{CONFLICT OF INTEREST}

None declared.

\section{REFERENCES}

1. Turk C, Knoll T, Petrik A, et al.: European Association of Urology, Guidelines on Urolithiasis. 2013.

2. Sountoulides P, Metaxa L, Cindolo L: Is computed tomography mandatory for the detection of residual stone fragments after percutaneous nephrolithotomy? J Endourol. 2013; 27: 1341-8.
3. Rosa M, Usai P, Miano R, Kim FJ, Finazzi Agrò E, Bove P, et al.: Recent finding and new technologies in nephrolitiasis: a review of the recent literature. BMC Urol. 2013; 13: 10.

4. Olbert P, Weber J, Hegele A, Varga Z, Heidenreich A, Hofmann $\mathrm{R}$ : Combining Lithoclast and ultrasound power in one device for percutaneous nephrolithotomy: in vitro results of a novel and highly effective technology. Urology. 2003; 61: 55-9; discussion 59.

5. Haupt G, Sabrodina N, Orlovski M, Haupt A, Krupin V, Engelmann $U$ : Endoscopic lithotripsy with a new device combining ultrasound and lithoclast. J Endourol. 2001; 15: 929-35.

6. Pietrow PK, Auge BK, Zhong P, Preminger GM: Clinical efficacy of a combination pneumatic and ultrasonic lithotrite. J Urol. 2003; 169: 1247-9.

7. Karakan T, Diri A, Hascicek AM, Ozgur BC, Ozcan S, Eroglu M: Comparison of ultrasonic and pneumatic intracorporeal lithotripsy techniques during percutaneous nephrolithotomy. ScientificWorldJournal. 2013; 2013: 604361.

8. Cho CO, Yu JH, Sung LH, Chung JY, Noh CH: Comparison of percutaneous nephrolithotomy using pneumatic lithotripsy (lithoclast $\AA$ ) alone or in combination with ultrasonic lithotripsy. Korean J Urol. 2010; 51: 783-7.

9. Auge BK, Lallas CD, Pietrow PK, Zhong P, Preminger GM: In vitro comparison of standard ultrasound and pneumatic lithotrites with a new combination intracorporeal lithotripsy device. Urology. 2002; 60: 28-32.

10. Pietrow PK, Auge BK, Zhong P, Preminger GM: Clinical efficacy of a combination pneumatic and ultrasonic lithotrite. J Urol. 2003; 169: 1247-9.

11. Tepeler A, Binbay M, Yuruk E, Sari E, Kaba M, Muslumanoglu AY, Tefekli A: Factors affecting the fluoroscopic screening time during percutaneous nephrolithotomy. J Endourol. 2009; 23: 1825-9.

12. Acar C, Cal C: Impact of Residual Fragments following Endourological Treatments in Renal Stones. Adv Urol. 2012; 2012: 813523.

13. Mercado A, Fernández MI, Recabal P, Fleck D, Ledezma R, Moya $F$, et al.: Immediate postoperative morbidity in patients with indwelling double-J stent versus overnight-externalized ureteral catheter after tubeless percutaneous nephrolithotomy: a prospective, randomized study. Urolithiasis. 2013; 41: 253-6.

14. Benson JS, Hart ST, Kadlec AO, Turk T: Small bore catheter drainage of pleural injury after percutaneous nephrolithotomy: feasibility and outcome from single large institution series. J Endourol. 2013; 2. [Epub ahead of print].

Correspondence address: Kursad Zengin

Bozok Universitesi Tip Fakultesi, Uygulama ve Arastirma Hastanesi, Uroloji Ana Bilim Dali, Yozgat, Turkey Fax: + $90354214-0612$ E-mail: kursadzengin@yahoo.com 